\title{
ROCHE INSTABILITY IN EJECTING STELLAR SYSTEMS
}

\author{
K.S.V.S. Narasimhan and S.M. Alladin \\ Centre of Advanced Study in Astronomy, \\ Osmania University, Hyderabad, India
}

\begin{abstract}
We consider a small stellar system ejected from the interior of a bigger one. The two systems are represented by unequal plummer models and energy changes in them are derived analytically under the impulse approximation. These are used to obtain the conditions for the Roche instability of the systems. For systems greatly differing in dimension, the density ratio of the two systems is a useful parameter for specifying the Roche 1 imit for the disruption of the smaller system and the mass ratio is a meaningful parameter for specifying the Roche limit for the disruption of the bigger system. Density ratios and mass ratios at Roche 1 imit are given for both systems for various scale-length ratios of the two syst ems.
\end{abstract}

\section{INTRODUCTION}

Of much interest in recent times has been the idea of ejection of matter from galaxies in various forms - gas (Gerola and Carnevali 1983, Fukunaga-Nakamuraand Toss 1989), radio jets (Yokosawa and Inoue 1985), quasars (Hutchings 1983, Narlikar and Das 1986, Burbidge and Hewitt 1989), black hol es (Kapoor 1985, Mikkola and Val tonen 1990) and stellar syst ems (Ambart sumian 1965, Arp 1972, 1986).

Saslaw, Valtonen and Asrseth (1974) discuss the gravitational slingshot mechanism for the ejection of an object from the centre of a galaxy, when three or more massive objects near the centre interact strongly. 
In the 1950's Ambartsumian suggested that galaxies ejected material from which new galaxies formed. Arp (1986) has drawn attention to observations which indicate that in the Virgo cluster of galaxies, the galaxy M 84 had originated as a protogalaxy within the bigger galaxy $M 87$ and had been ejected out along the 1 ine of the jet. $X$-ray observations show that $M 84$ is moving almost exactly along the same line from M 87. In the Stephen's Quintet, one of the five galaxies has a very different $r$ ed shift from those of the rest and could have been ejected from the group. Our galaxy and $M 31$ were probably ejected from the same initial protogalaxy. Thus the ejection of a smaller system for a bigger one is of much astronomical interest.

In this paper we consider the ejection of a smaller stellar system by a bigger stellar system and determine the increase in energy of the constituent stars in each system on account of tidal forces. Initially both the systems are assumed to be in virial equilibrium. Using the Jacobi' criterion of stability, namely, that a system is unstable if its total energy is positive, we deduce the conditions under which each of the two systems becomes unstable.

\section{STELLAR VELOCITY PERTUR BATIONS}

Let $M_{1}$ and $M_{2}$ be the masses of the bigger system (ejector) and the smaller system (ejected) respectively. We consider both systems to be spherically symmetric configurations of density distribution represented by plummer model, namely,

$$
\rho_{i}(r)=\frac{3 M_{i} \alpha_{i}^{2}}{4 \pi} \frac{1}{\left(r^{2}+\alpha_{i}^{2}\right)^{5 / 2}}, i=1,2
$$

where

$$
\alpha_{i}=\left(\frac{3 M_{i}}{4 \pi\left(p_{i}\right)_{i}}\right)^{1 / 3}
$$

$\rho_{i}$ being the central density. Let $\alpha_{12}=\frac{\alpha_{1}}{\alpha_{2}}$ be the scale length ratio and let $\alpha_{1} \geq \alpha_{2}$.

Consider the tidal effects of $M_{1}$ and $M_{2}$. Let the centre of $M_{2}$ be the origin of the coordinate system. Let $\vec{r}$, and $\vec{r}$ denote the position of the centre of $M_{1}$ and of a representative star in $M_{2}$ respectively and let $\vec{r}^{\prime \prime}=\vec{r}^{\prime}-\vec{r}$. The motion of the star in $M_{2}$ is given by 


$$
\ddot{r}=-\nabla \Phi_{2}+\nabla " \Phi_{1}-\frac{1}{M_{2}} \nabla \cdot w
$$

and $\Phi$ and $W$ represent the potential and the interaction potential energy given by

$$
\begin{aligned}
\Phi_{i}(r) & =-\frac{G M_{i}}{\left(\alpha_{i}^{2}+r^{2}\right)^{1 / 2}}, i=1,2 \\
W(r) & =-\frac{G M_{1} M_{2}}{\left(\alpha^{2}+r^{2}\right)^{1 / 2}}
\end{aligned}
$$

$\alpha$ is a complicated function of $\alpha_{1}$ and $\alpha_{2}$. We shall make the approximation $\alpha^{2}=\alpha_{1}^{2}+\alpha_{2}^{2}$ as in Aarseth and Fall (1980) in carrying out subsequent integration. The first term on the right hand side in equation (3) represents the gravitational attraction of $M_{2}$ on the star while the remaining $t$ wo terms together give the tidal acceleration $\vec{f}_{T}$ due to the ejector $M_{1}$.

Let $M_{2}$ be ejected from the centre of $M_{1}$ along the $z$-axis with velocity $\mathrm{V} \geq \mathrm{v}_{\text {par }}$ where $\mathrm{v}_{\text {par }}$ is parabolic velocity of escape of the two systems. We obtain the change in the velocity of the star by integrating $\vec{f}_{T}$ over time using the impul se approximation,i.e. neglecting the motion of the star during the ejection. The impulse approximation gives reasonable est imates for the energy changes in stellary systems moving with relative speeds equal to or greater than the parabolic speed (Alladin and Narasimhan 1982).

Following the analysis as in Zafarullah, Narasimhan and Sastry (1983), we get the following expressions for the velocity changes of the star perpendicular to and parallel to the direction of ejection:

$$
\begin{aligned}
& \Delta V_{1}=\left[\left(\Delta V_{x}\right)^{2}+\left(\Delta V_{y}\right)^{2}\right]^{1 / 2}=\frac{G_{1} \tilde{w}}{V\left(\tilde{w}^{2}+\alpha_{1}^{2}\right)} \\
& \Delta V_{11}=\Delta V_{z}=\frac{G M_{1}}{V}\left[\frac{1}{\left(w^{2}+\alpha_{1}^{2}\right)^{1 / 2}}-\frac{1}{\left(\alpha_{1}^{2}+\alpha_{2}^{2}\right)^{1 / 2}}\right]
\end{aligned}
$$

where $\tilde{w}=\left(x^{2}+y^{2}\right)^{1 / 2}$. 


\section{ENERGY CHANGES IN EJECTION}

The increment in the kinetic energy $\Delta T_{\text {g }}$ due to the $t i-$ daleffects $M_{1}$ which in the impulse approximation is the same as increment in the total energy $\mathrm{\Delta U}_{2}$ is obtained from

$$
\Delta_{2}=\int_{0}^{\infty}\left[\left\{\Delta V_{\perp}(\tilde{w})\right\}^{2}+\left\{\Delta v_{11}(\tilde{w})\right\}^{2}\right] d M_{2}
$$

We follow the analysis given in Atmed (1979) and Binney and Tremaine (1987) for integrating over the mass. The integration is carried out analytically and obtain

$$
\begin{aligned}
\Delta_{2} & \equiv\left(\Delta U_{1}\right)_{2}+\left(\Delta U_{11}\right)_{2} \\
& =\frac{G^{2} M_{1}^{2} M_{2} B\left(\alpha_{12}\right)}{2 v^{2} \alpha_{1}^{2}}+\frac{G^{2} M_{1}^{2} \mathrm{M}_{2}}{2 v^{2} \alpha_{1}^{2}}\left[\mathrm{C}\left(\alpha_{12}\right)+D\left(\alpha_{12}\right)+E\left(\alpha_{12}\right)\right] \\
& \equiv \frac{G^{2} M_{1}^{2} M_{2}}{2 v^{2} \alpha_{1}^{2}} H\left(\alpha_{12}\right)
\end{aligned}
$$

where $B\left(\alpha_{12}\right)=\frac{2 \alpha_{12}^{2}}{\alpha_{12}^{2}}\left[\frac{\alpha_{12}^{2}+1}{\alpha_{12}^{2}}\right.$ in $\left.\alpha_{12}-1\right]$

$$
\begin{aligned}
C\left(\alpha_{12}\right) & =\frac{\alpha_{12}^{2}}{\alpha_{12}^{2}}\left[1-\frac{2}{\alpha_{12}^{2}-1} \ln \alpha_{12}\right] \\
D\left(\alpha_{12}\right) & =\frac{\alpha_{12}^{2}}{\alpha_{12}^{2}} \\
-E\left(\alpha_{12}\right) & =\frac{2 \alpha_{12}^{2}}{\alpha_{12}^{2}-1} \cdot \frac{1}{\left(\alpha_{12}^{2}+1\right)^{1 / 2}}\left[\frac{\ln \left\{\alpha_{12}+\left(\alpha_{12}^{2}-1\right)^{1 / 2}\right\}}{\alpha_{12}^{2}-1}-\alpha_{12}\right]
\end{aligned}
$$

Table I gives $B\left(\alpha_{12}\right)$ and $H\left(\alpha_{12}\right)$. 
Table I: The Functions $B\left(\alpha_{12}\right)$ and $H\left(\alpha_{12}\right)$

\begin{tabular}{lcc}
$\alpha_{12}$ & $B\left(\alpha_{12}\right)$ & $H\left(\alpha_{12}\right)$ \\
\hline 1.0 & $1.67(-1)$ & $2.24(-1)$ \\
1.5 & $1.56(-1)$ & $2.04(-1)$ \\
2.0 & $1.38(-1)$ & $1.77(-1)$ \\
3.0 & $1.05(-1)$ & $1.30(-1)$ \\
4.0 & $8.12(-2)$ & $9.80(-2)$ \\
5.0 & $6.45(-2)$ & $7.64(-2)$ \\
10 & $2.75(-2)$ & $3.10(-2)$ \\
20 & $1.01(-2)$ & $1.10(-2)$ \\
50 & $2.33(-3)$ & $2.48(-3)$ \\
100 & $7.21(-4)$ & $7.60(-4)$ \\
500 & $4.17(-5)$ & $4.36(-5)$ \\
1000 & $1.16(-5)$ & $1.20(-5)$ \\
\hline
\end{tabular}

(n) $=10^{\bar{n}}$

by

The self-gravitational potential energy of $M_{2}$ is given

$$
\Omega=-\frac{3 \pi}{32} \frac{G_{2}^{2}}{a_{2}}=2 U_{2} \text { by vivial theorem }
$$

whence the fractional increase in the binding energy of $\mathrm{M}_{2}$ is given by

$$
\mu_{2} \equiv \frac{\Delta U_{2}}{\mid U_{2} T}=\frac{32}{3 \pi} \frac{\mathrm{GM}_{1}^{2} \alpha_{2}}{M_{2} \mathrm{~V}^{2} \alpha_{1}^{2}} \cdot \mathrm{H}\left(\alpha_{12}\right)
$$

Following the analysis in Ahned (1979) and Alladin and Narasiman (1986), equation (11) can be written in the form

$$
\mu_{2}=\frac{16}{3 \pi} \frac{1}{M_{21}\left(1+M_{21}\right)} \frac{\alpha_{21} H\left(\alpha_{12}\right)}{A\left(\alpha_{12}\right)}\left(\frac{V_{\text {par }}}{V}\right)^{2}
$$

where $M_{21}=\frac{M_{2}}{M_{1}}, \alpha_{21}=\frac{\alpha_{2}}{\alpha_{1}} ; V_{p a r}$ is the parabolic speed of escape of the two systems of ejection. The function $A\left(\alpha_{12}\right)$ is given in Almed (1979).

Similarly we obtain for the change in the binding energy of the ejector $M_{1}$ :

$$
\begin{aligned}
\mu_{1} \equiv \frac{\Delta \mathrm{U}_{1}}{\mid \tilde{U}_{1} T} & =\frac{32}{3 \pi} \frac{\mathrm{GM}_{2}^{2} \alpha_{1}}{\mathrm{M}_{1} \mathrm{~V}^{2} \alpha_{2}} \mathrm{H}\left(\alpha_{12}\right) \\
& =\frac{16}{3 \pi} \frac{1}{\mathrm{M}_{12}\left(1+\mathrm{M}_{12}\right)} \frac{\alpha_{12} \mathrm{H}\left(\alpha_{12}\right)}{\mathrm{A}\left(\alpha_{12}\right)}\left(\frac{\mathrm{V}_{\mathrm{par}}}{\mathrm{V}}\right)^{2}
\end{aligned}
$$




\section{ROCHE LIMITS IN EJECTION}

According to Jacobi's criterion of stability, a stellar system is unstable if its total energy is positive. This implies that $\mu \equiv \frac{\Delta U}{\mid U T}>1$. However, the numerical experiments by Miller (1986) indicate that a satellite system is in danger of disintegration if $\mu>2$. When $\mu 1$ ies between 1 and 2 many stars remain bound. We shall adopt the criterion $\mu=1$ for obtaining the Roche limits in ejecting systems.

Table II gives the mass ratios and density ratios at the Roche 1 imits for various values of $\alpha_{12} \cdot \mu_{1}=1$ gives the limit for the disruption of the bigger system and $\mu_{2}=1 \mathrm{gi-}$ gives the corresponding limit for the smaller system. For a given $\alpha_{12}$, the bigger system is unstable for the values of $M_{12}=\frac{M_{1}}{M_{2}}$ or equivalent $1 \mathrm{y} \quad \rho_{12}=\rho_{1} / \rho_{2}$ smaller than the tabular value and the smaller system is unstable for values of $M_{12}$ Table II: Mass ratios and density ratios at Roche limit when $\mathrm{V}=\mathrm{v}_{\text {par }}$

\begin{tabular}{|c|c|c|c|c|}
\hline & \multicolumn{4}{|c|}{ Roche Limit for the Disruption of } \\
\hline & Bigger & Syst em & Smaller & System \\
\hline$\alpha_{12}$ & $M_{12}$ & $\rho_{12}$ & $\mathrm{M}_{12}$ & $\rho_{12}$ \\
\hline $\begin{array}{l}1.0 \\
1.5 \\
2.0 \\
3.0 \\
4.0 \\
5.0 \\
10 \\
20 \\
50 \\
100 \\
500 \\
1000\end{array}$ & $\begin{array}{l}0.45 \\
0.67 \\
0.84 \\
1.10 \\
1.29 \\
1.44 \\
1.88 \\
2.29 \\
2.79 \\
3.13 \\
3.83 \\
4.04\end{array}$ & $\begin{array}{l}4.50(-1) \\
1.99(-1) \\
1.05(-1) \\
4.74(-2) \\
2.02(-2) \\
1.15(-2) \\
1.88(-3) \\
2.86(-4) \\
2.23(-5) \\
3.13(-6) \\
3.06(-7) \\
4.04(-9)\end{array}$ & $\begin{array}{l}2.22 \\
3.83 \\
6.03 \\
1.25(+1) \\
2.27(+1) \\
3.66(+1) \\
1.87(+2) \\
1.07(+2) \\
1.19(+4) \\
7.76(+4) \\
6.78(+6) \\
4.92(+7)\end{array}$ & $\begin{array}{l}2.22 \\
1.13 \\
7.60(-1) \\
4.64(-1) \\
3.53(-1) \\
2.13(-1) \\
1.85(-1) \\
1.34(-1) \\
9.52(-1) \\
7.76(-2) \\
5.42(-2) \\
4.92(-2)\end{array}$ \\
\hline
\end{tabular}

$$
(n)=10^{n}
$$

or $\rho_{12}$ greater than the tabular value. It may be noted from the table that for the bigger system the range in $M_{12}$ is considerably smaller than the range in $\rho_{12}$ while for the smaller 
system the reverse bolds. Hence for specifying the instability of the bigger system, the mass ratio is a useful parameter; while for specifying the instability of the smaller system, the density ratio is a meaningful parameter.

The dependence of $M_{12}$ on $\alpha_{21}$ when $V=v_{\text {par }}$ and $\mu_{1}=1$ can be expressed as

$$
\mathrm{M}_{12}=(2.6)(0.13)^{\alpha} 21
$$

Using the Chi-square Test, we find the fit to be good both at $5 \%$ and $1 \%$ levels of significance. Further as $\alpha_{12} \rightarrow \infty$, $M_{12}+2.6 \sim 3$. We therefore, infer that a smaller system will generally disrupt a bigger system at $v=v_{\text {par }}$ if its mass is greater than one-third the mass of the bigger.

The dependence of $\left(\rho_{1}\right)_{12}$ on $M_{21}$ when $V=V_{\text {par }}$ and $\mu_{2}=1$ is of the form

$$
\left(\rho_{i}\right)_{12}=0.16(10)^{3 M_{21}}
$$

The $f i t$ is again found to be good. As $M_{12}+\infty,\left(\rho_{i 1}\right)_{12} \rightarrow 0.16$ $\sim \frac{1}{7}$. This indicates that the bigger system will generally make the smaller unstable if its density is greater than oneseventh of the density of the smaller at $V=V_{\text {par }}$.

A similar analysis yields the values $1 / 2$ and $1 / 3$ for the disintegration of the bigger and smaller systems respectively if $\mathrm{Miller} ' s(1986)$ criterion $\mu=2$ is used.

\section{COMPARISON WITH COLL IDING STELLAR SYSTEMS}

Energy transfer in head -0 collisions of galaxies were studied analytically by Toomre (1977) and Ahmed (1979). Nara simhan and Alladin (1986) derived the conditions for Roche instability for head on collisions of stellar systems. A comparison of the results for the colliding systems with those obtained here for ejecting systems indicates that the fractional increase in the binding energy of a system in the case of a collision is about 3 times that in ejection. The corresponding Roche 1 imits in the case of a collision are 1/6 and $1 / 25$.

In the case of a collision $\Delta V_{11}=0$. In ejection $\Delta V_{11}$ is small but is not zero.

\section{CONCLUSION}

The anal ytical treatment of energy changes under the impul se approximation leads to the following results: (i) The 
energy change in an ejection is about $1 / 3$ of that in a complete collision. (ii) For stellar systems differing widely in dimensions the disruption of the smaller system in an ejection is conveniently specified by the density ratio of the two systems and the disruption of the larger by the mass ratio. (iii) When the velocity of ejection is equal to the parabolic velocity of escape of the pair, the smaller system is generally unstable if its density is less than about 7 times the density of the bigger and the bigger is unstable if its mass is less than about 3 times the mass of the smaller.

It is desirable to test the accuracy of the predictions of the analytical formulae presented here by numerical experiments.

\section{REFERENCES}

[1] Asrseth, S.J. and Fall, S.M. (1980), Astrophys. J.236, 43 .

[2] Almed, F. (1979), Astrophys. Space Sc. 60,493.

[3] Alladin, S.M. and Narasimhan, K.S.V.S. (1982), Phys.Rep. 92,339 .

[4] Ambartsumian, V.A. (1965), in "The Structure and Evolution of Galaxies", Interscience, New York, p.1.

[5] Arp, H.C. (1972), in "External Galaxies and Quasi Stel lar Objects", ed. D.S.Evans, Dordrecht: D.Reidel .

[6] Arp, H.C. (1986), J .A strophys. A stron. 7,71.

[7] Binney, J. and Tremaine, S. (1987), "Galactic Dynamics", Princeton University Press, p.439.

[8] Burbidge, G. and Hewitt, A. (1989), in IAU Symp. 134: "Active Galactic Nuclei", ed. D.E. Osterbrock and J.S. Miller, Kuwer Academic Publishers, p.562.

[9] Fukunaga-Nakumars, A. a nd Toss, M. (1989), Publ .Astron. Soc. Japan, 41, 953 .

[10] Gerola, H. and Carnevali, P. (1983), Astrophys.J. 268, L.75.

[11] Hutchings, J.B. (1983), Publ . Astron. Soc. Pacif ic, 95, 799.

[12] Kapoor, R.C. (1985), Astrophys. Space Sc. 112,347.

[13] Mikkola, S. and Val tonen, M.J. (1990), A strophys.J.348, 412.

[14] Milier, R.M. (1986), Astron. Astrophys. 167,41.

[15] Narasimhan, K.S.V.S. and Alladin, S.M.(1986), Astrophys. Space Sc. 128,307 .

[16] Narlikar, J.V. and Das, P.K.(1986), A strophys.J.24 0, 407.

[17] Saslaw,W.C., Valtonen,M.J. and Aarseth, S.J.(1974), Astrophys.J .190, 253.

[18] Toomre, A.(1977), in "The Evolution of Galaxies and Stellar Populations", ed.B.M.Tinsley and R.B.Larson, Yale University Press, p.401.

[19] Yoko sowa, M. and Inoue, M. (1985), Publ .A stron. Soc .Japa n, 37 , 655.

[20] Zafarullah, S.N., Narasimhan, K. S.V.S. and Sasstry, K.S. (1983), Bul1.Astr. Soc. India, 11, 145 . 